\title{
Molecular persistent luminescence imaging with porphyrin derivatives for ultrasensitive image-guided cancer surgery and drug screening
}

Xingchen Duan ${ }^{1+}$, Guoqiang Zhang ${ }^{1+}$, Shenglu $\mathrm{Ji}^{1}$, Yiming Zhang ${ }^{1}$, Jun $\mathrm{Li}^{1}$, Hanlin $\mathrm{Ou}^{1}$, Zhiyuan $\mathrm{Gao}^{1}$, Guangxue Feng ${ }^{2}$, Dan Ding ${ }^{1 *}$

${ }^{1}$ Frontiers Science Center for Cell Responses, State Key Laboratory of Medicinal Chemical Biology, Key Laboratory of Bioactive Materials, Ministry of Education, and College of Life Sciences, Nankai University, Tianjin 300071, China.

${ }^{2}$ AIE Institute, State Key Laboratory of Luminescent Materials and Devices, Guangdong Provincial Key Laboratory of Luminescence from Molecular Aggregates, School of Materials Science and Engineering, South China University of Technology, Guangzhou 510640, China

*Corresponding author. Email: dingd@ @nankai.edu.cn

${ }^{+}$These authors contributed equally to this work.

Persistent luminescence without excitation light and tissue autofluorescence interference holds great promise for in vivo imaging and sensing. However, the availability of persistent luminescence materials is largely limited by potential toxicity, instability, short-wavelength emissions, and poor clinical potential for currently available ones. Here we report a series of porphyrin derivatives with near-infrared (NIR) persistent luminescence for image-guided cancer surgery and drug screening. These porphyrin derivatives showed NIR persistent luminescence over $760 \mathrm{~nm}$ after cessation of excitation light or upon interaction with peroxynitrite $\left(\mathrm{ONOO}^{-}\right)$, and a plausible mechanism of ordered oxidation of vinylene bond is proposed. Through molecular engineering with adaptive peptides bearing the functions of $\beta$ sheet-formatting and cancer cell targeting, the resultant Ppa-FFGYSA supermolecular probe showed enhanced photoacoustic and persistent luminescence signals, facilitating preoperative photoacoustic tumor identification and intraoperative persistent luminescence image-guided tumor resection with superior signal-to-background ratio. In addition, the activated persistent luminescence in recognition of $\mathrm{ONOO}^{-}$also permits the specific monitoring of neutrophil infiltration and screening of immunogenic cell death (ICD) drugs with high sensitivity and specificity. 


\section{Introduction}

Optical imaging represents an indispensable tool in biological and biomedical research ${ }^{1-3}$. Its unique advantages of high spatial-temporal resolution, superior sensitivity, and fast acquisition time permit the diagnostic imaging, real-time image-guided surgery, and monitoring of disease progresses and therapeutic responses, etc ${ }^{4-8}$. Many fluorophores (such as indocyanine green, fluorescein sodium, Cetuximab-IRDye800CW) have been applied in clinical practice or under clinical evaluation ${ }^{9-11}$, and great efforts have also been put to develop second near-infrared (NIR-II, 1000-1700 nm) fluorophores and upconversion nanomaterials to improve the imaging depth and sensitivity ${ }^{12-18}$. However, most of the widely used fluorophores requires real-time excitation light to produce fluorescence signals, which inevitably generate tissue autofluorescence background that compromises signal-to-background ratios (SBRs) and imaging quality ${ }^{19}, 20$.

Persistent luminescence (PL) is a distinct luminescent process that can continuously release photons after cessation of excitation light or without real-time light excitation ${ }^{21,22}$. Such light-excitation-free PL circumvents the autofluorescence noise interference, promising for in vivo optical imaging applications with high SBRs and specificity ${ }^{23-25}$. However, the availability of PL probes for clinical translation are hampered by their own demerits. For example, Cerenkov probes face biosafety issues raised from their radioisotopic nature ${ }^{26,27}$. Inorganic PL nanocrystals consist of toxic rare-earth heavy metals accompanied with difficulty in surface modification ${ }^{28-30}$. Semiconducting polymers and Schaap's dioxetane based ones are new emerging organic PL materials whose biocompatibility and functionality are largely improved, but are still limited by short-wavelength emission and high hydrophobicity ${ }^{31,32}$. Construction of nanoparticles with energy transfer pairs could redshift PL to NIR region but increases the complexity and reduces the reproducibility, limiting their potential for clinical translation ${ }^{33-35}$. To date, the development of long NIR emissive PL molecular probes with excellent biocompatibility and ease of functionalization for high-contrast deep tissue imaging remains challenging.

Here, we report porphyrin derivatives with PL for intraoperative image-guided surgery and drug screening. Particularly, we aimed to demonstrate that the ability of clinically used porphyrin derivatives to show PL after cessation of light sources or upon interaction with $\mathrm{ONOO}^{-}$for the first time. Molecular screening found that Ppa show promising PL at $760 \mathrm{~nm}$ that can last over $60 \mathrm{~min}$ after cessation of excitation light, and with a high specificity to be activated by peroxynitrite ( $\mathrm{ONOO}^{-}$) over other reactive oxygen and nitrogen species (RONS). A plausible mechanism with ordered oxidation of vinylene bond was proposed for such PL phenomenon. By molecular engineering with adaptive $\beta$-sheet-formatting peptides, the PpaFFGYSA supermolecular aggregate shows enhanced photoacoustic and PL signals that are 
beneficial for preoperative photoacoustic tumor locating and intraoperative PL image-guided tumor resection with ultrahigh SBRs. As $\mathrm{ONOO}^{-}$is the hallmark of neutrophil infiltration and immunogenic cell death (ICD), Ppa-FFGYSA serves as a smart activable molecular probe for screening of ICD drugs in living mice with high specificity, which outperformed traditional immunofluorescence staining approaches.

\section{Results}

\section{Phenomenon and mechanistic study of PL}

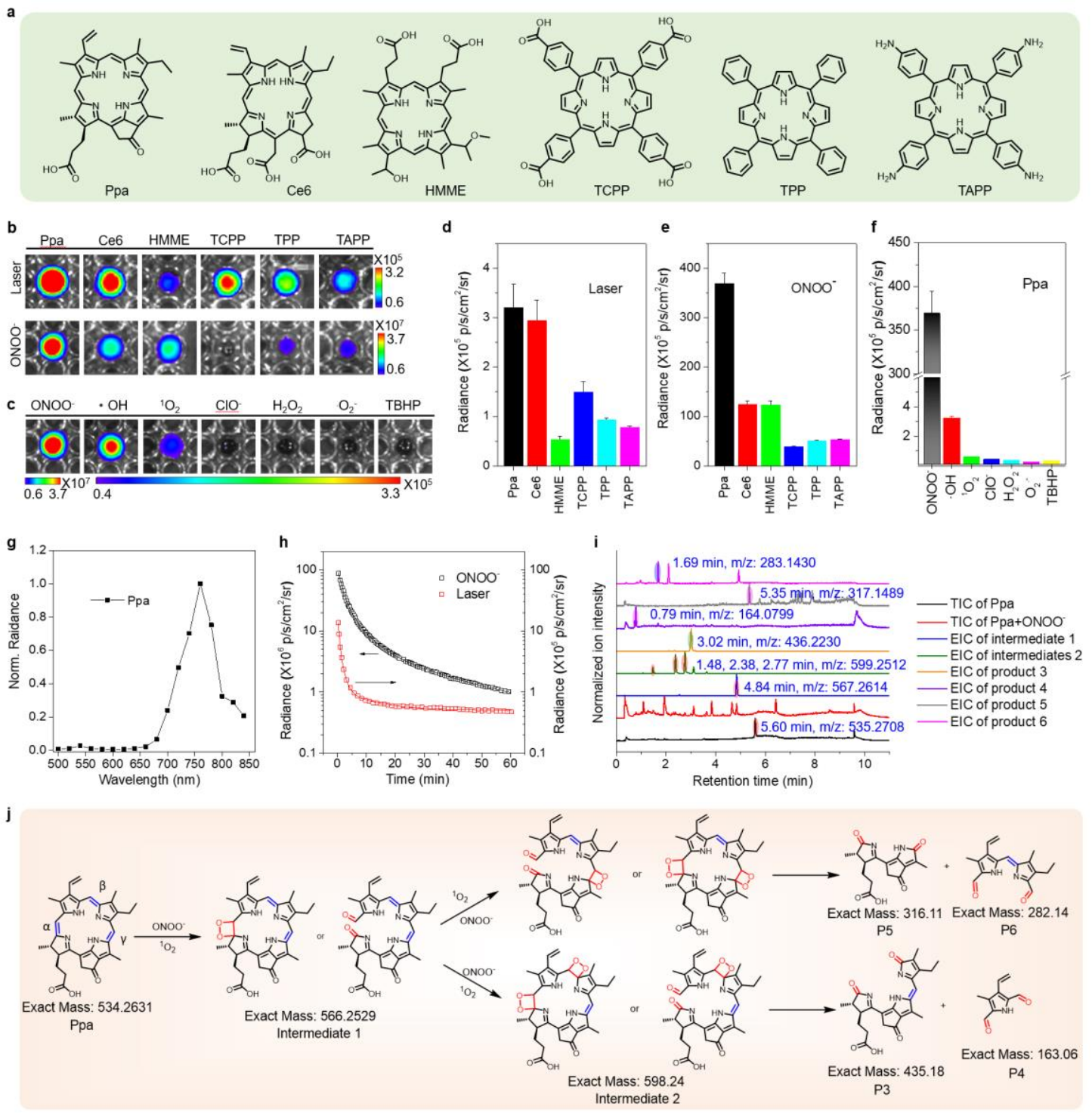

Fig. 1 Characterization and mechanistic study of PL of porphyrin derivatives. (a) Chemical structures of the porphyrin derivatives (Ppa, Ce6, HMME, TCPP, TPP, and TAPP). (b) PL images of Ppa, Ce6, HMME, TCPP, TPP, and TAPP $(1 \mathrm{mg} / \mathrm{ml}, 50 \mu \mathrm{L})$ in $1 \times$ PBS buffer $(\mathrm{pH}=7.4)$ after cessation of light pre-irradiation $\left(660 \mathrm{~nm}, 1.4 \mathrm{~W} / \mathrm{cm}^{2}, 2 \mathrm{~min}\right)$ or the addition of $\mathrm{ONOO}^{-}(80 \mu \mathrm{M})$. (c) PL images of 
Ppa after addition of different RONS, including $\mathrm{ONOO}^{-},{ }^{1} \mathrm{O}_{2}, \cdot \mathrm{OH}, \mathrm{O}_{2}{ }^{--}, \mathrm{ClO}^{-}, \mathrm{H}_{2} \mathrm{O}_{2}, \mathrm{TBHP}(80 \mu \mathrm{M})$. (df) The quantification of corresponding PL intensities in b and c. (g) Normalized PL spectrum of Ppa obtained by collecting signals every $20 \mathrm{~nm}$ on IVIS after adding ONOO- $^{-}(80 \mu \mathrm{M})$. (h) Decay of PL signal of Ppa $(1 \mathrm{mg} / \mathrm{mL})$ over time at room temperature after addition of $\mathrm{ONOO}^{-}(80 \mu \mathrm{M})$ or cessation of light pre-irradiation ( $\left.660 \mathrm{~nm}, 1.4 \mathrm{~W} / \mathrm{cm}^{2}, 2 \mathrm{~min}\right)$. The PL images were acquired for $60 \mathrm{~s}$ with an open filter. (i) The total ion chromatography (TIC) of Ppa before and after addition of $\mathrm{ONOO}^{-}$as well as the extracted ion chromatography (EIC) of intermediates and products, insets are their retention time and corresponding mass. (j) The proposed mechanism for PL of Ppa. Error bars mean \pm SD ( $n=3)$.

To molecularly screen the capability of PL, six porphyrin derivatives (Ppa, Ce6, HMME, TCPP, TPP, and TAPP) with different but similar molecular structures (Fig. 1a) were selected and dissolved in $1 \times$ phosphate buffer (PBS) with the aid of amphiphilic block copolymer (DSPE-PEG 2000 ) as the surfactant. These porphyrin molecules show typical Soret band at 400 $\mathrm{nm}$ and $\mathrm{Q}$ bands in the range of $450-650 \mathrm{~nm}$, with fluorescence maxima ranging from 650 to $750 \mathrm{~nm}$ (Supplementary Fig. 1a,b). The singlet oxygen $\left({ }^{1} \mathrm{O}_{2}\right)$ generation ability of these porphyrin derivatives was evaluated by 9,10-anthracenediyl-bis(methylene dimalonic acid) (ABDA) under light irradiation. The decreased ABDA absorbance at $400 \mathrm{~nm}$ indicates that ${ }^{1} \mathrm{O}_{2}$ is produced by porphyrin derivatives under light illumination (Supplementary Fig. 2). The PL signals of porphyrin molecules were collected with an IVIS imaging system (Fig. 1b, c). Upon cessation of pre-irradiation laser $\left(660 \mathrm{~nm}, 1.4 \mathrm{~W} / \mathrm{cm}^{2}, 2 \mathrm{~min}\right)$, all the porphyrin molecules showed remarkable PL, where Ppa and C6 yielded the highest signals (Fig. 1b, d). ONOO- with high oxidation ability was further selected to evaluate whether these porphyrin derivatives could be chemically excited. As shown in Fig. 1b, addition of $\mathrm{ONOO}^{-}$could readily light-up the PL of these porphyrin solutions whereas Ppa gave the highest PL signal (Fig. 1d). Furthermore, the PL signal activated by $\mathrm{ONOO}^{-}$is much higher than that activated by light preirradiation (Fig. 1d, e).

Ppa was further selected to evaluate the PL selectivity towards other RONS, including ${ }^{1} \mathrm{O}_{2}, \cdot \mathrm{OH}, \mathrm{O}_{2}{ }^{-\cdot}, \mathrm{ClO}^{-}, \mathrm{H}_{2} \mathrm{O}_{2}$, and tert-butyl hydroperoxide (TBHP). Interestingly, ONOOrepresents a much more efficient PL initiator over other RONS, outperforming by 2-3 orders of magnitude (Fig. 1c, f). Such ONOO-activated PL behaviors were also observed for the other five porphyrin molecules (Supplementary Fig. 3). The PL signals of Ppa showed a very good linear correlation with $\mathrm{ONOO}^{-}$concentrations with a limit of detection (LOD) of $64.2 \mathrm{nM}$, calculated from $3 \sigma / \mathrm{k}$ ( $\sigma$ is the standard deviation of blank tests and $\mathrm{k}$ is the slope of working curve) (Supplementary Fig. 4). The PL peak of Ppa locates at $\sim 760 \mathrm{~nm}$ in the physiological $1 \times$ PBS buffer (Fig. 1g, Supplementary Fig. 5), much longer than the most existing single fluorophore based PL probes. The PL signal show the decay half-lives of about 2-15 minutes (Fig. 1h, Supplementary Figs. 6,7), indicating the photon releasing a long-lasting process, 
which are long enough for imaging and drug screening purposes.

The PL mechanism of Ppa was then investigated via the ultra-high performance liquid chromatography-high resolution mass spectrometry (UPLC-HRMS) analysis with ONOO$^{-}$as the initiator. Ppa itself showed a retention time of $5.60 \mathrm{~min}$ (Fig. 1i, black line and Supplementary Fig. 8, $\left.\mathrm{M}_{(\mathrm{Ppa})}+1: 535.2708\right)$, which disappeared after exposure to $\mathrm{ONOO}^{-}$, with the emergence of multiple molecular ion peaks at other retention time (Fig. 1i, red line), indicating of fragmentation of Ppa by $\mathrm{ONOO}^{-}$oxidation. These fragments were further extracted for UPLC-HRMS analysis. The formation of unstable dioxetane intermediates via the $\pi^{2}-\pi^{2}$ cycloaddition towards vinylene bond $(\mathrm{C}=\mathrm{C})$ is reported to be responsive for the PL of semiconducting polymers ${ }^{32}$. The similar Ppa-dioxetane intermediates were also found after addition of $\mathrm{ONOO}^{-}$to Ppa solution, which were at the UPLC retention time of 1.48, 2.38, 2.77 and 4.84 min, respectively (Fig. 1i, blue and green lines; Supplementary Fig. 9, $\mathrm{M}_{(\mathrm{Ppa})}+32+1$ : 567.2614; Supplementary Fig. 10, $\mathrm{M}_{(\mathrm{ppa})}+64+1$ : 599.2512). Based on the cycloaddition of oxygen atoms, the intermediate 1 at 4.84 min represents the first step of $\pi^{2}-\pi^{2}$ cycloaddition of one $\mathrm{C}=\mathrm{C}$ bond $\left(\mathrm{M}_{(\mathrm{Ppa})}+32+1\right)$, while the intermediates 2 at 1.48, 2.38 and $2.77 \mathrm{~min}$ are the products of the oxidation of the following second $\mathrm{C}=\mathrm{C}$ bond $\left(\mathrm{M}_{(\mathrm{ppa})}+64+1\right)$. The break of two $\mathrm{C}=\mathrm{C}$ bonds leads to the decomposition of Ppa. The final decomposed fragments were further identified to reveal the exact oxidation orders of $\alpha, \beta$ and $\gamma \mathrm{C}=\mathrm{C}$ bonds on the cyclic ring of Ppa. Four final fragments with two possible pairs of P3/P4 (m/z of 436.2230 and 164.0799) (Fig. 1i, orange and purple line, Supplementary Fig. 11), and P5/P6 (m/z of 317.1489 and 283.1430) Fig. 1i, pink and grey line, Supplementary Fig.12) were found. The P3/P4 pair resulted from the oxidation of $\alpha$ and $\beta \mathrm{C}=\mathrm{C}$ bonds, while $\mathrm{P} 5 / \mathrm{P} 6$ pair was the product of the oxidation of $\alpha$ and $\gamma \mathrm{C}=\mathrm{C}$ bonds, respectively. Both are the results of the second oxidation step of intermediates 2 . The shared $\alpha \mathrm{C}=\mathrm{C}$ bond was thus identified to be oxidized in the first step. Hence, a plausible mechanism for PL of Ppa was proposed (Fig. 1j). The $\mathrm{ONOO}^{-}$will firstly oxidize $\alpha \mathrm{C}=\mathrm{C}$ double bond followed by the simultaneous oxidation of $\beta$ or $\gamma \mathrm{C}=\mathrm{C}$ double to form unstable Ppadioxetane intermediates. The degradation of unstable Ppa-dioxetane into Ppa-aldehyde releases photons for such PL, and the self-generated ${ }^{1} \mathrm{O}_{2}$ ensures the sustainable photon releasing after cessation of light irradiation.

\section{Construction of supermolecular probe}



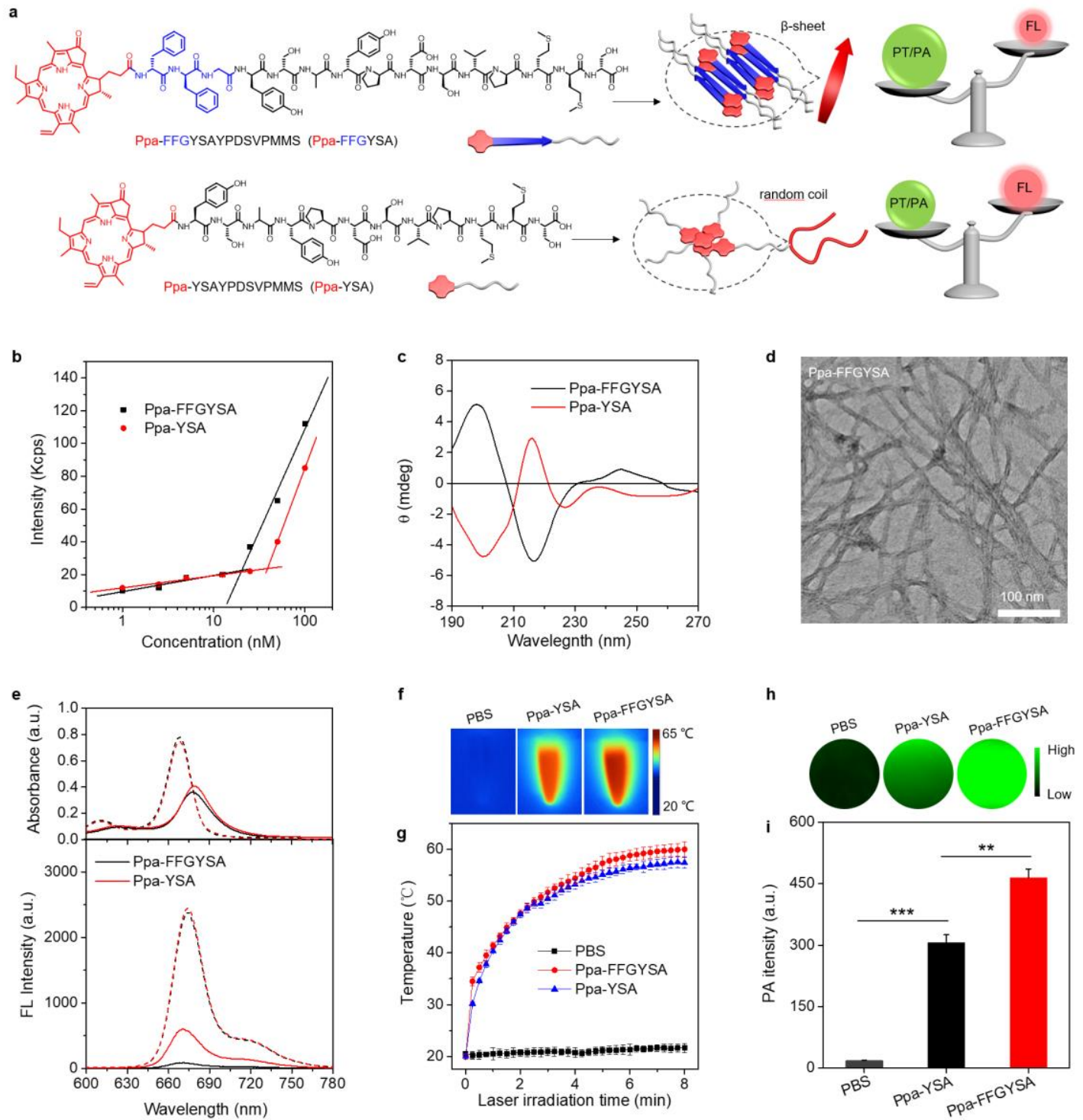

Fig. 2 Construction of Ppa-FFGYSA supermolecular probe. (a) Chemical structures of Ppa-FFGYSA, Ppa-YSA and the schematic illustration of the excited energy balance in photophysics of these supermolecular probes. (FL: fluorescence, PT/PA: photothermal/photoacoustic). (b) Determination of CMC values of Ppa-FFGYSA and Ppa-YSA from DLS. (c) CD spectra of Ppa-FFGYSA and Ppa-YSA supermolecular aggregates in $1 \times$ PBS buffer at a concentration of $1 \mathrm{mM}$. (d) TEM image of PpaFFGYSA supermolecular aggregates. (e) The UV-vis absorption and fluorescence profiles of PpaFFGYSA and Ppa-YSA in monomer (dissolved in DMSO, dashed lines) and in aggregates (dissolved in $1 \times$ PBS buffer with 5\% DMSO, solid lines). (f) IR images of PBS buffer, Ppa-FFGYSA and Ppa-YSA aggregates $\left(50 \mu \mathrm{M}\right.$ in $1 \times$ PBS buffer) after 8 min laser irradiation $\left(660 \mathrm{~nm}, 1.4 \mathrm{~W} / \mathrm{cm}^{2}\right)$. (g) Timedependent temperature elevation of Ppa-FFGYSA and Ppa-YSA aggregates (50 $\mu \mathrm{M}$ in PBS) under 660 $\mathrm{nm}$ laser irradiation $\left(1.4 \mathrm{~W} / \mathrm{cm}^{2}\right.$ ). (h) Photoacoustic images of PBS buffer, Ppa-FFG-YSA and Ppa-YSA acquired on MOST and (i) the corresponding photoacoustic signal intensities. Error bars mean \pm SD (n =3). ** $P<0.01$, and *** $P<0.001$.

To afford porphyrin molecules the adaptive function for optical imaging, Ppa was selected 
and conjugated with the smart supermolecular peptide FFGYSAYPDSVPMMS (FFGYSA) to obtain Ppa-FFGYSA conjugate (Supplementary Figs. 13-19). FFG serves as the $\beta$-sheet formation group, ${ }^{36-38}$ and YSA is a targeting peptide towards $\mathrm{EphA}_{2}$ clusters on tumor cell membrane (Fig. 2a). Such $\beta$-sheet supermolecular aggregate was designed to enhance the photoacoustic performance at expense of fluorescence signal through strong $\pi$ - $\pi$ stacking effects to facilitate preoperative tumor identification via photoacoustic imaging. Ppa-YSA without $\beta$-sheet formation group was also synthesized as a control. Ppa-FFGYSA and Ppa-YSA showed very similar UV-vis absorption and fluorescence spectra as that of Ppa in DMSO solution, suggesting that peptide conjugation leads to minimal changes to the optical properties of Ppa in the monomer state (Supplementary Fig. 20). However, the situation become quite different for their supermolecular aggregates in PBS buffer. Ppa-FFGYSA and Ppa-YSA in phosphate buffered saline (PBS) showed critical micelle concentrations (CMCs) of 19.2 and $40.1 \mu \mathrm{M}$, respectively (Fig. 2b). The circular dichroism (CD) spectra collected at the concentrations above CMC revealed a typical $\beta$-sheet structure (a negative peak at $217 \mathrm{~nm}$ and a positive peak at $198 \mathrm{~nm}$ ) for Ppa-FFGYSA, while random coil aggregates (a negative peak at $200 \mathrm{~nm}$ and a small positive peak at around $216 \mathrm{~nm}$ ) for Ppa-YSA, respectively (Fig. 2c). Transmission electron microscopy (TEM) images further confirmed their different aggregation behaviors and morphologies, where regularly and orderly nanofibrous structures were observed for Ppa-FFGYSA and irregularly curled morphologies for Ppa-YSA (Fig. 2d, Supplementary Fig. 21).

Both Ppa-FFGYSA and Ppa-YSA aggregates in PBS buffer showed decreased fluorescence as compared to Ppa aggregates, indicating the strong $\pi-\pi$ stacking effect for the former two supermolecular aggregates (Fig. 2e) ${ }^{39,40}$. The nearly diminished fluorescence for Ppa-FFGYSA further suggests the closely ordered molecular packing caused by $\beta$-sheet formatting FFG peptide segments. Similar phenomenon was observed when increasing water fractions in Ppa-FFGYSA containing water/DMSO mixture (Supplementary Fig. 22). CD spectra confirmed the $\pi-\pi$ molecular stacking for Ppa-FFGYSA, where the bisignate CD signals in $\mathrm{Q}$ band indicate the exciton coupling of the transition dipole moments ${ }^{41-43}$ (Supplementary Fig. 23). In addition, the ${ }^{1} \mathrm{O}_{2}$ generation abilities for these two peptide supermolecular aggregate were well-retained in PBS solution, where Ppa-YSA aggregate showed slightly better ${ }^{1} \mathrm{O}_{2}$ generation efficiency (Supplementary Fig. 24). According to Jablonski diagram, reducing fluorescence could redirect more excited energy to ground through non-radiative dissipation pathways, leading to more generation of heat, beneficial for photothermy and photoacoustic imaging ${ }^{44}$. The photothermal properties of Ppa-FFGYSA and PPa-YSA supermolecular aggregates in PBS solution were further evaluated. Ppa-FFGYSA showed a higher temperature elevation over Ppa-YSA under $660 \mathrm{~nm}$ laser irradiation, with a steady-state temperature difference of $2.6^{\circ} \mathrm{C}$, which is large enough to induce significant enhancement of photoacoustic signal (Fig. 2f,g). Photoacoustic signal was collected on a small-animal opt-acoustic 
tomography (MOST, iTheraMedical, Germany). The PA spectrum $(680-900 \mathrm{~nm})$ was in consistence with the absorption profile of Ppa, confirming the origin of photoacoustic signal from Ppa (Supplementary Fig. 25a). Ppa-FFGYSA showed a higher PA intensity than that of Ppa-YSA at all concentrations as expected with an enhancement factor of 1.6. (Fig. 2h,i, Supplementary Fig. 25b).

\section{Tissue penetration of Ppa-FFGYSA supermolecular probe}
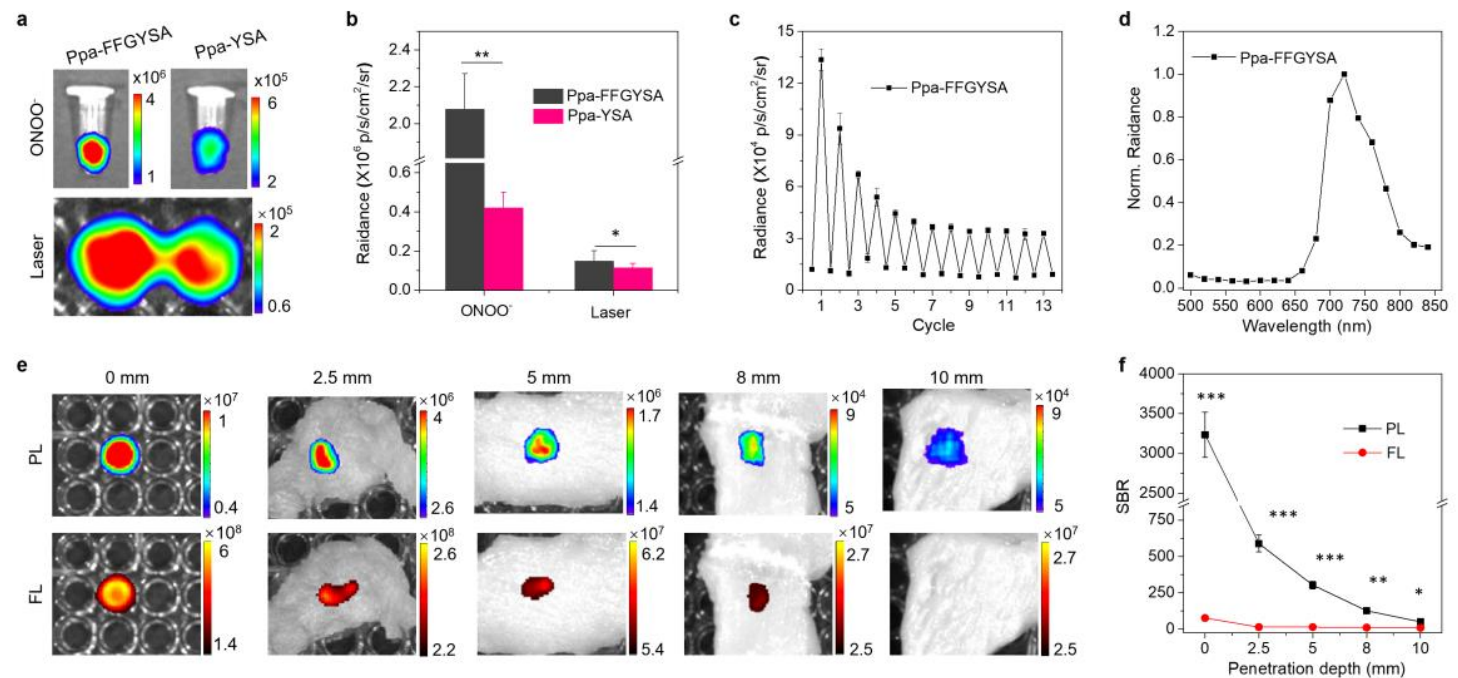

Fig. 3 PL characterization of Ppa-FFGYSA supermolecular probe. (a) PL images of Ppa-FFGYSA and Ppa-YSA in PBS solution (500 $\mu \mathrm{M}$, PBS: DMSO=95:5, v/v, PH=7.4) after addition of $\mathrm{ONOO}^{-}$(top row) or cessation of light pre-irradiation (bottom row). (b) Quantification of the PL intensities in a. (c) The PL signal changes of Ppa-FFGYSA in PBS buffer after repeated laser activation for 13 cycles. (d) PL spectrum of Ppa-FFGYSA in $1 \times$ PBS buffer obtained by collecting the PL signal every $20 \mathrm{~nm}$ on IVIS after the addition of $\mathrm{ONOO}^{-}$. (e) PL and fluorescence (FL) images of Ppa-FFGYSA (500 $\left.\mu \mathrm{M}\right)$ solutions through chicken tissues of different thicknesses, PL images were acquired immediately after adding $\mathrm{ONOO}^{-}(80 \mu \mathrm{M})$ to Ppa-FFGYSA PBS solution. (f) SBRs for PL or FL images as a function of tissue depth. Error bars, mean $\pm \mathrm{SD}(\mathrm{n}=3)$. ** $P<0.01$, and $* * * P<0.001$.

The suitability of Ppa-FFGYSA for PL imaging was firstly evaluated with light pre-irradiation. The optical light irradiation for activating PL was firstly optimized to be $1.4 \mathrm{~W} / \mathrm{cm}^{2}$ for $2 \mathrm{~min}$ (Supplementary Fig. 25). The retained ${ }^{1} \mathrm{O}_{2}$ generation ability (Supplementary Fig. 24) ensures the self-sustained PL after cessation of light irradiation. Ppa-FFGYSA showed 1.29-fold stronger PL intensity than that of Ppa-YSA after light pre-irradiation (Fig. 3a, b). Higher PL signals were observed when using $\mathrm{ONOO}^{-}$as the initiator, and the difference between PpaFFGYSA and Ppa-YSA was much higher (4.95-fold higher for Ppa-FFGYSA) than that of PL signals activated by light pre-irradiation. The enhanced PL for Ppa-FFGYSA is attributed to its $\beta$-sheet structure. The peripheral arrangement of Ppa-FFGYSA with more surface area makes Ppa much easier for $\mathrm{ONOO}^{-}$contact to oxidize the vinylene bond. In addition, a relative higher 
temperature $\left(37^{\circ} \mathrm{C}\right)$ could further enhance the PL signal (Supplementary Fig. 27), suggesting the higher photothermal performance of Ppa-FFGYSA (Fig. 2g) shall also contribute to its enhanced PL over Ppa-YSA aggregate. The PL signal could last over 60 min (Supplementary Fig. 28), and it could be repeatedly activated by light irradiation over 10 cycles (Fig. 3c), which provides sufficient operation window and reliability for in vivo PL imaging and image-guided surgery.

Ppa-FFGYSA and Ppa-YSA show similar PL spectra as Ppa, the emission maximum at $740 \mathrm{~nm}$ confers deeper tissue penetrating properties at NIR region (Fig. 3d, Supplementary Fig. 29). The imaging depth and sensitivity for Ppa-FFGYSA was studied by increasing the thickness of chicken tissue pieces placed on top of the samples. The fluorescence signal was acquired with real-time $660 \mathrm{~nm}$ laser excitation, and the PL was induced by addition of ONOO'. Without real-time excitation interference, PL showed a superior SBR of 3235.3, which was 42.5-fold higher than that of fluorescence (SBR of 76.2) without chicken tissue. Both PL and fluorescence signals of Ppa-FFGYSA decreased when increasing the thickness of the chicken tissues (Fig. 3e). PL signal was still detectable at a thickness of $10 \mathrm{~mm}$ while fluorescence signal become invisible due to the poor penetration ability of the excitation light and the high background signal. As PL possesses a very low background noise, its SBR was still 49.3 at a thickness of $10 \mathrm{~mm}$, whereas fluorescence of Ppa-FFGYSA was close to the tissue autofluorescence background (Fig. 3f). Therefore, PL could eliminate the tissue autofluorescence noise and circumvent photon scattering of excitation light, beneficial for higher imaging depth and SBRs for in vivo imaging.

The feasibility of Ppa-FFGYSA for biological applications was confirmed by its excellent biocompatibility on breast cancer cell (4T1) with overexpressed EphA2 receptors and normal cell (smooth muscle cell, SMC, with low EphA2 receptor expression) (Supplementary Fig. 30). The targeting ability was then validated by confocal laser scanning microscopy (CLSM) imaging (Supplementary Fig. 31). Co-staining with anti-EphA2 antibody confirmed its specificity towards EphA2 receptor (Supplementary Fig. 32), which was further validated by blocking experiment with excessive free YSA to block the EphA2 receptor on the surface of 4T1 cells (Supplementary Fig. 33). The targeting selectivity of Ppa-FFGYSA to EphA2 overexpressed cancer cells was further confirmed via SMC cell with low expressing EphA2 receptor as negative control cells (Supplementary Fig. 34). Collectively, Ppa-FFGYSA with high PL signal and excellent cancer cell specificity permits the sensitive cancer imaging and diagnosis applications.

\section{PL image-guided tumor surgery}


a
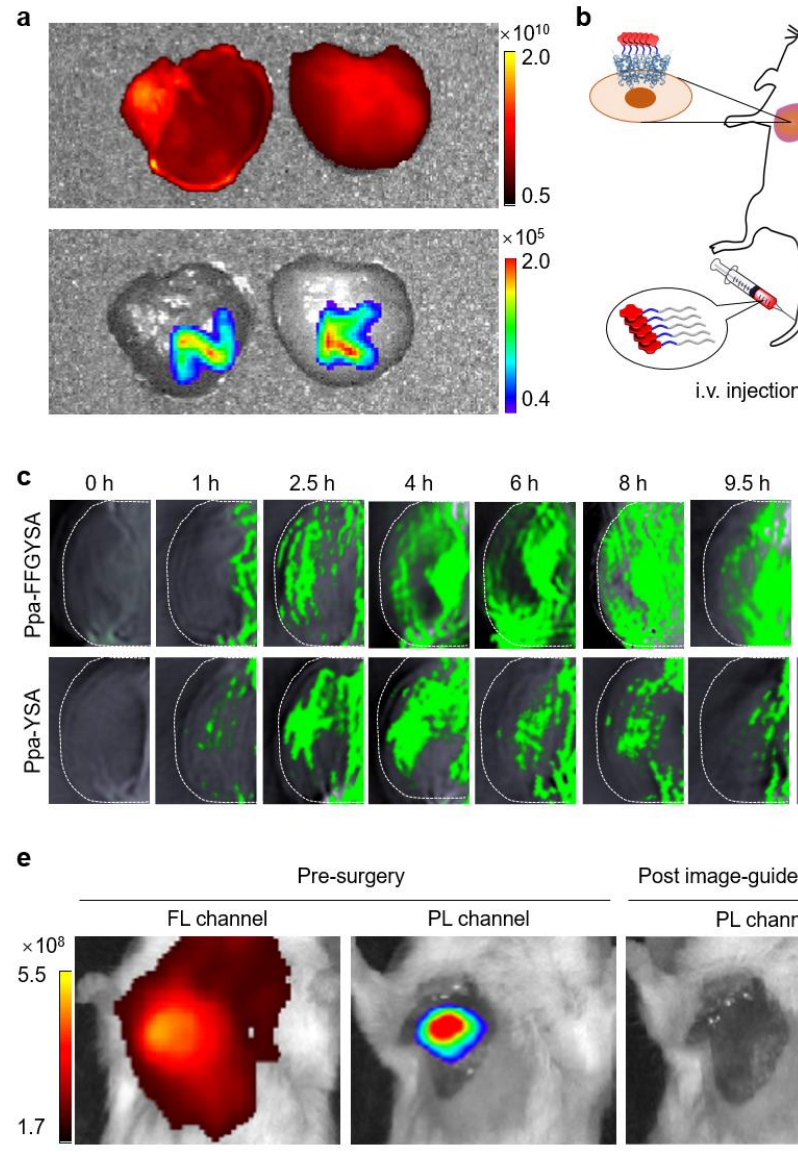

Pre-surgery

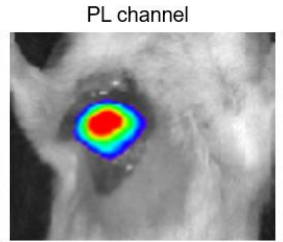

Post image-guided surgery
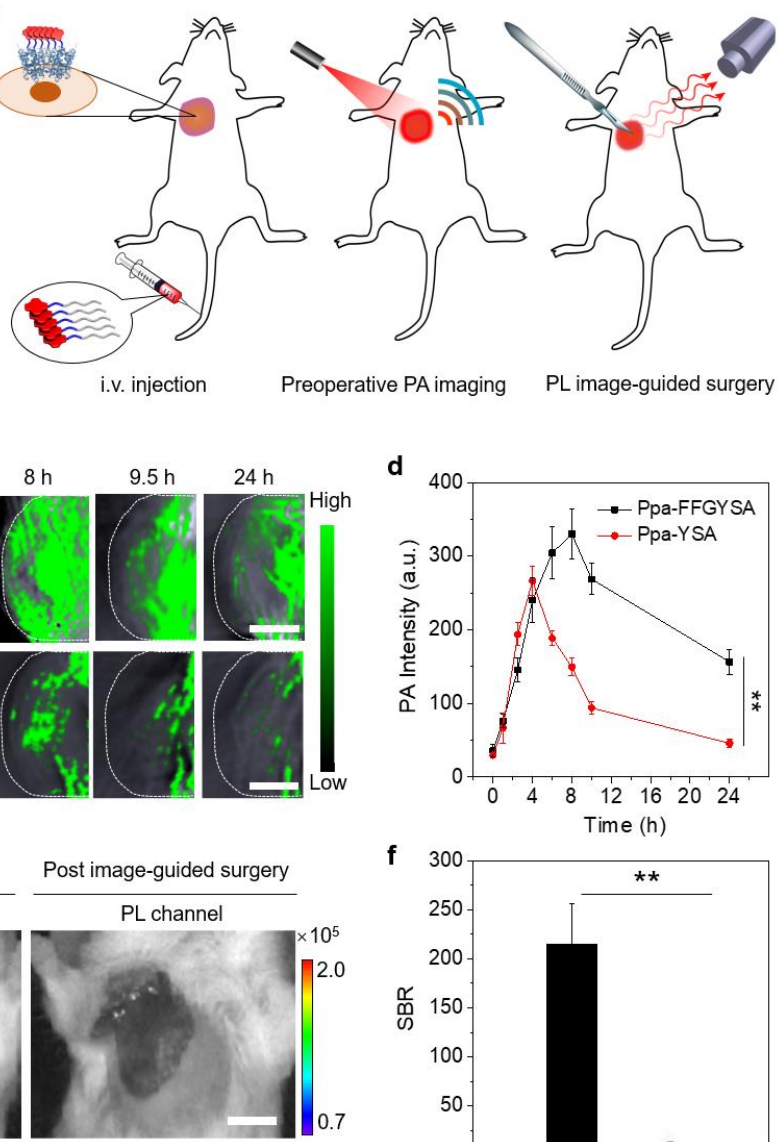

i.v. injection
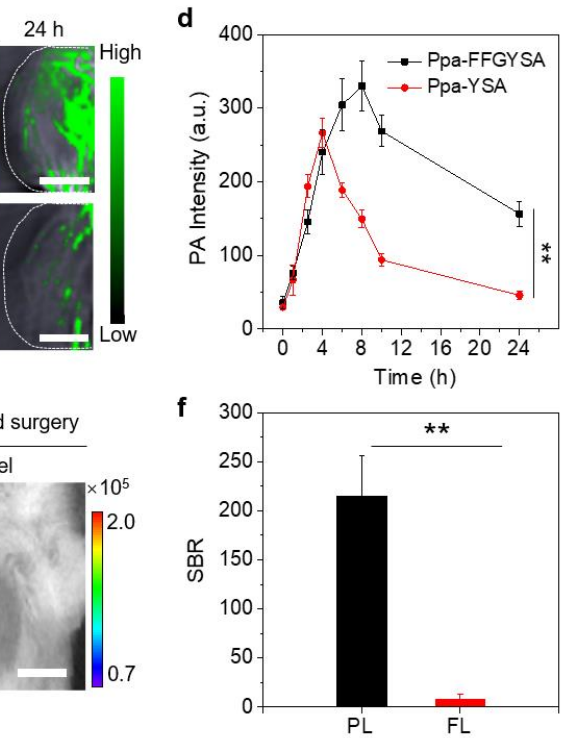

g
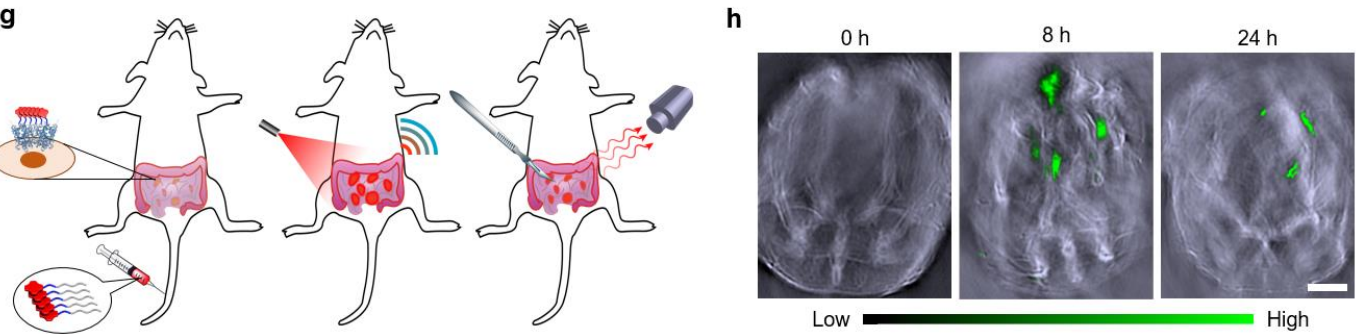

i.v. injection Preoperative PA imaging PL image-guided surgery

i
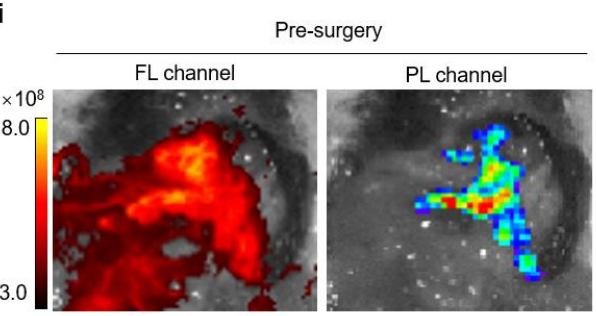

Post image-guided surgery
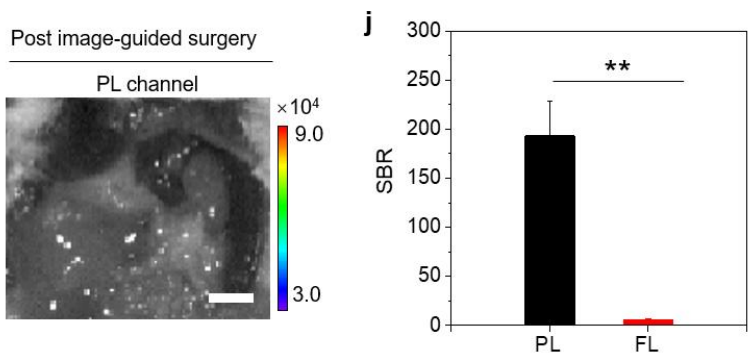

Fig. 4 Preoperative photoacoustic imaging and intraoperative PL-image guided cancer surgery. (a) $\mathrm{PL}$ and FL images of isolated livers at $1 \mathrm{~h}$ after injection of Ppa-FFGYSA through the tail vein. Livers were engraved with "N" and "K", and the PL was activated by laser pre-irradiation $\left(660 \mathrm{~nm}, 1.4 \mathrm{~W} / \mathrm{cm}^{2}\right.$, $2 \mathrm{~min}$ ). (b) Schematic illustration of Ppa-FFGYSA for preoperative photoacoustic imaging and intraoperative PL image-guided surgery on orthotopic 4T1 mammary tumor mouse model, PA: photoacoustic. (c) Representative photoacoustic images of tumor sites after intravenously injection of Ppa-FFGYSA and Ppa-YSA (500 $\mu$ M, PBS: DMSO=95:5, v/v) into orthotopic mouse breast cancer 
model, respectively. Scale bars, $2 \mathrm{~mm}$. (d) Quantification of photoacoustic intensities in tumor site at designated time points after probe injection. (e) Representative FL and PL images of mouse tumors before and after PL image-guided surgery. Scale bars, $5 \mathrm{~mm}$. Tumor resection was performed at $8 \mathrm{~h}$ post Ppa-FFGYSA (500 $\mu$ M, PBS:DMSO=95:5, v/v) injection. (f) Calculated SBRs of PL and FL image of tumor sites before surgery in e. (g) Schematic illustration of Ppa-FFGYSA for preoperative photoacoustic imaging and intraoperative PL image-guided surgery on peritoneal carcinomatosis-bearing mice. (h) Representative cross-sectional photoacoustic images of the mouse tumor site with peritoneal carcinoma before $(0 \mathrm{~h})$ and at $8 \mathrm{~h}$ and $24 \mathrm{~h}$ post intravenous injection of Ppa-FFGYSA. Scale bars, $1 \mathrm{~cm}$. (i) Representative FL and PL images of the peritoneal cavity after opening the abdomen (pre-surgery), and FL image of the peritoneal cavity after surgery guided by PL signals from Ppa-FFGYSA. Scale bars, 5 mm. (j) The SBRs of FL and PL images in i before surgery. Error bars, mean $\pm \mathrm{SD}(\mathrm{n}=3)$. ** $P<0.01$, and $* * * P<0.001$.

The in vivo biosafety of Ppa-FFGYSA and Ppa-YSA was firstly confirmed by H\&E staining of important normal organs as well as blood chemistry examinations after intravenous injection (Supplementary Figs. 35-37). To test whether PL could be activated in physiological condition, livers were harvested after intravenous injection of Ppa-FFGYSA, and engraved with "N" and "K" letters on the surface. After cessation of excitation light, engraved " $N$ " and "K" showed distinguishable PL signal from background while fluorescence signal covered all the liver tissues under real-time light excitation, proving the superior SBRs and imaging sensitivity brought by PL (Fig. 4a). The PL image-guided tumor surgery was firstly tested with an orthotopic breast cancer model built with 4T1 cells (Fig. 4b). As photoacoustic imaging could provide deeper information about tumors at the expense of imaging resolution ${ }^{45}$, the good photoacoustic properties of Ppa-FFGYSA enable preoperatively locating tumor site by MOST before tumor resection surgery. Ppa-FFGYSA was intravenously injected into 4T1 tumor bearing mice via the tail vein, and Ppa-YSA was also administrated into another group of mice for comparison. Fig. 4c shows the time-elapsed in vivo photoacoustic imaging. The photoacoustic signals at tumor site gradually increased for both groups, which reached the maximum at $4 \mathrm{~h}$ for Ppa-YSA and at $8 \mathrm{~h}$ for Ppa-FFGYSA, respectively (Fig. 4c, d), followed by gradually declining. Ppa-FFGYSA showed a better photoacoustic tumor identification over Ppa-YSA, which should be attributed to the cooperation of higher photoacoustic signal, longer blood circulation and better tumor retention of the nanofibrous structured Ppa-FFGYSA ${ }^{46}$. Such PA imaging provides important surgical planning information for subsequent tumor resection ${ }^{47}$.

It requires fast accessing all tumor masses without any residual tumor nodules left for cancer surgery $^{2,9}$. PL molecular probes with high cancer specificity and minimized background tissue autofluorescence interference appear to be the ideal candidates for image-guided surgery. With the tumor information provided by preoperative photoacoustic imaging, the PL imageguided surgical oncology treatment was performed at $8 \mathrm{~h}$ post tail vein injection of Ppa- 
FFGYSA. The tumor was irradiated with a $660 \mathrm{~nm}$ laser to activate PL. After cessation of excitation laser, the solid tumor and its contours showed bright PL signal that is distinguishable from surrounding healthy tissues. As there is no interference from tissue autofluorescence and excitation light, PL image gave a very high SBR of 215.1, which was 26.9 times higher than that of fluorescence image (SBR of 8.0) (Fig. 4f). Such high SBR and image contrast enable the complete tumor removal under PL image guidance (Fig. 4e), which was double confirmed by H\&E staining (Supplementary Fig. 38).

A peritoneal carcinomatosis-bearing mouse model was also established to verify the capability of Ppa-FFGYSA for PL image-guided resection of small tumor nodules (Fig. 4g). Photoacoustic signal from Ppa-FFGYSA revealed the existence of tumors at the abdominal cavity of the mice, which also provided preoperative information about tumor location (Fig. 4h). PL image-guided tumor resection was then performed at $24 \mathrm{~h}$ post-injection to exclude undesirable potential signal interference from the intestine. Different from fluorescence image that cannot avoid tissue atuofluorescence interference, the noise-free PL could clearly delineate tumors from surrounding healthy organs in such a complicated peritoneal cavity environment (Fig. 4i, j). Benefited from such high SBR (193.1), submillimeter tumor nodules could be successfully resected under the PL guidance. Ex vivo H\&E staining as well as fluorescence and PL images of the excised peritoneum tumors and small intestine tumors further verified the accuracy of the surgery (Supplementary Fig. 39). Therefore, our Ppa-FFGYSA supermolecular aggregate could largely improve tumor resection outcomes by preoperative PA imaging localization and intraoperative PL image-guided surgery, which significantly reduces tumor recurrence possibility.

\section{PL screening of ICD drugs}


a

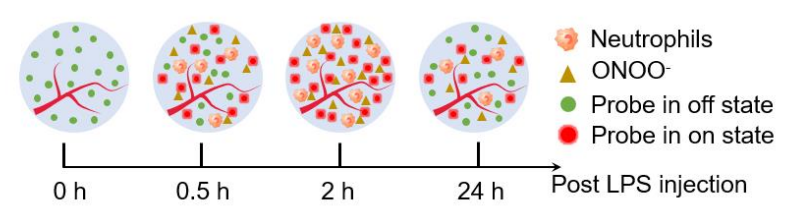

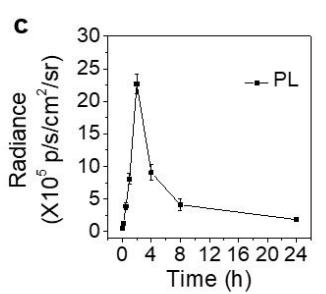

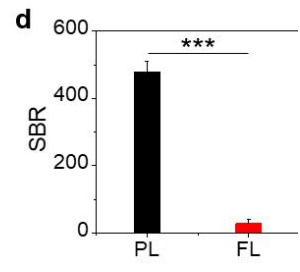

b

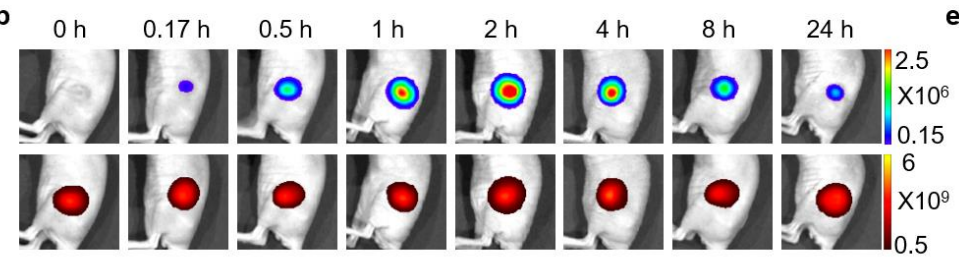

e $\quad 0.5 \mathrm{~h}$

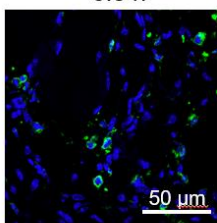

$2 \mathrm{~h}$

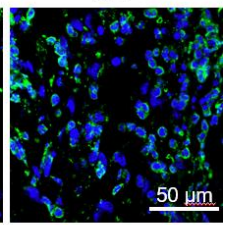

f
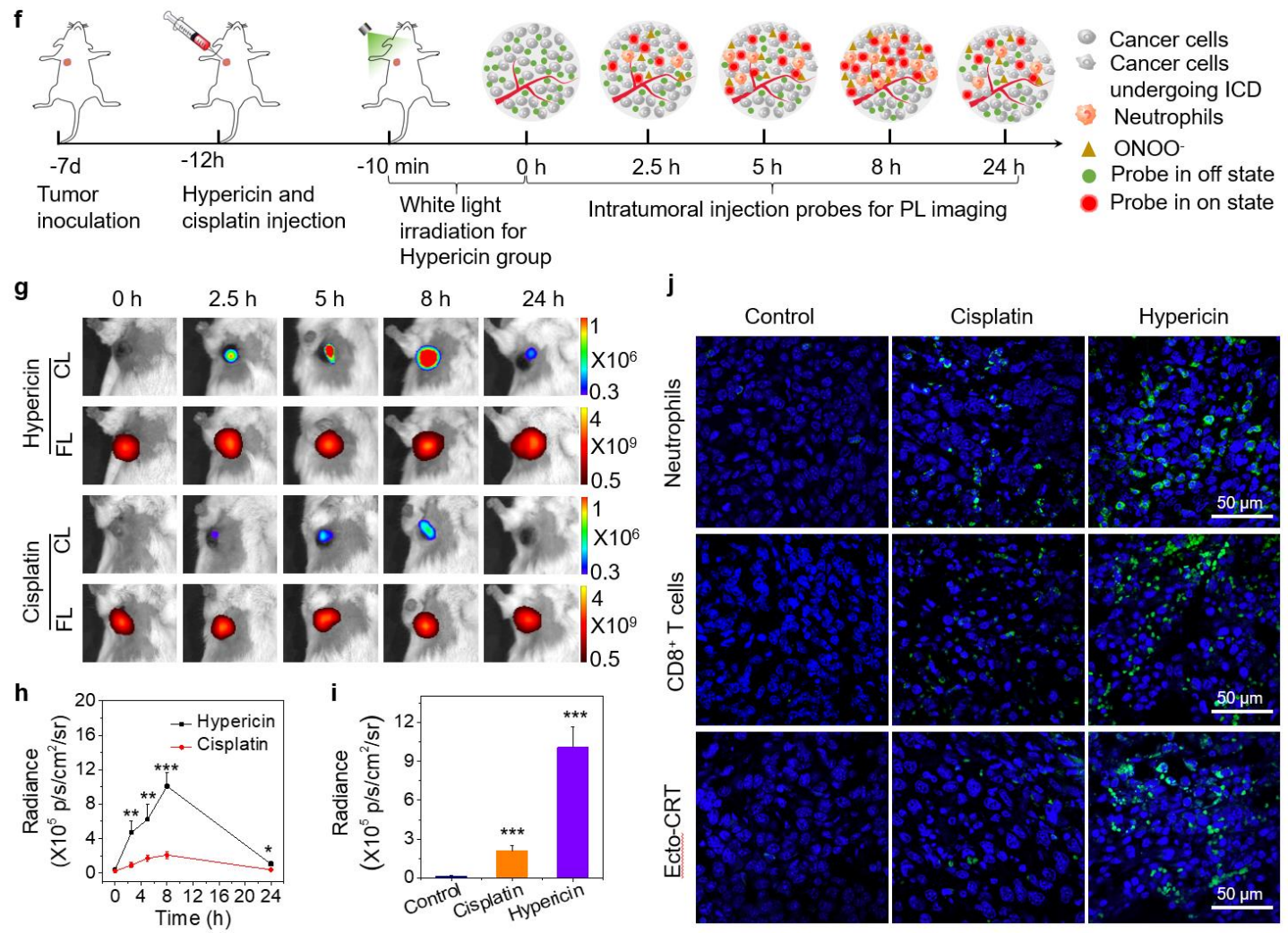

Fig 5. PL screening of ICD drugs with Ppa-FFGYSA. (a) Schematic illustration of monitoring neutrophil infiltration and $\mathrm{ONOO}^{-}$level with PL from Ppa-FFGYSA. (b) Representative PL and FL images of LPS injection sites at predesignated time post LPS injection. Images was acquired immediately after Ppa-FFGYSA injection, where PL images were acquired for $60 \mathrm{~s}$ at an open filter and the FL images were acquired at $720 \mathrm{~nm}$ under real-time excitation at $675 \mathrm{~nm}$. (c) PLintensity changes in b as a function of post LPS injection time. (d) SBRs at $2 \mathrm{~h}$ based on the CL and FL signals in (b). (e) Representative CLSM images of immunofluorescent staining of neutrophils in LPS injection site at $0.5 \mathrm{~h}$ and $2 \mathrm{~h}$ post LPS injection. Green: neutrophils; blue: nuclei. (f) Schematic illustration of PL monitoring of ICD druginduced tumor microenvironment transformation from "cold" to "heat". The PDT treatment part of tumor would experience neutrophil infiltration and $\mathrm{ONOO}^{-}$generation, leading to activation of PL signal of the injected Ppa-FFGYSA probe. (g) Representative time-dependent PL and FL images of the 4T1 tumorbearing mice with tumor received hypericin or cisplatin treatment. Ppa-FFGYSA probe was in situ injected into the whole tumors of mice in each group at predesignated time intervals. (h) Quantitative 
analysis of PL intensities at tumor sites as a function of time based on images in g. (i) Quantitative comparison of activated PL intensities between control, hypericin and cisplatin groups. (j) Representative confocal images indicating the levels of infiltrated neutrophils, infiltrated $\mathrm{CD} 8^{+} \mathrm{T}$ cells and ecto-CRT at the drug administration part of tumor in each treatment group and quantitative analysis of immunofluorescence intensities based on the typical immunostaining images. Error bars, mean \pm SD (n $=3)$. ** $P<0.01$, and $* * * P<0.001$.

Identification and quantification of early biomarkers or theranostic response is crucial for screening of new drugs ${ }^{8,48}$. Smart molecular probes that undergo specific luminescence lightup upon recognizing such biomarkers or responses offer high contrast and real-time information on pathological conditions at the molecular level ${ }^{49-51}$. The development of immunogenic cell death (ICD) therapy drugs is crucial for immunotherapy as they can turns the tumor microenvironment from "cold" to "hot" by increasing infiltration of immune cells especially neutrophils and $\mathrm{CD}^{+} \mathrm{T}$ cells, making tumor more liable to immunotherapy ${ }^{52,53}$. Neutrophil infiltration usually occurs at the first stage of ICD therapy ${ }^{54}$, which induces massive generation of RONS especially $\mathrm{ONOO}^{-}$(in vivo generation could be as high as $50-100 \times 10^{6} \mathrm{M} / \mathrm{min}$ ) ${ }^{55}, 56$. The specificity of our Ppa-FFGYSA probes to show PL toward ONOO- makes itself the ideal candidate for real-time monitoring of neutrophil infiltration and activation of ICD therapy.

A neutrophil infiltration model was established by subcutaneous inoculation of lipopolysaccharide (LPS) $(50 \mu \mathrm{g}, 25 \mu \mathrm{L})$ into mice (Fig. 5a). The Ppa-FFGYSA supermolecular probe $(500 \mu \mathrm{M}$ dissolved in $1 \times$ PBS buffer, $\mathrm{pH}=7.4)$ was further injected at predesignated time points, immediately followed by IVIS imaging. There was no obvious change in fluorescence signal at each time points, which indicates a consistent amount of probe injection to provide reasonable comparison of PL signal changes. The PL signal was turned on at LPS site at $0.17 \mathrm{~h}$ post LPS inoculation, suggesting the generation of $\mathrm{ONOO}^{-}$as a response of neutrophil infiltration. The PL signal gradually inclined and reached the maximum at $2 \mathrm{~h}$, which then declined thereafter within tested time period (Fig. 5b, c). Noteworthy, PL image yielded a very high SBR of 478.3 at $2 \mathrm{~h}$ post LPS injection (Fig. 5d), and such high sensitivity and SBR prove the great merits of activable molecular PL probes for dynamic monitoring biological responses. Immunofluorescence staining of neutrophils revealed a nearly identical trends of neutrophils infiltration as to the activated PL signals (Fig. 5e, Supplementary Fig. 40). To eliminate the pH influence, the PL signals of Ppa-FFGYSA probe in PBS buffers with different $\mathrm{pH}$ conditions ( $\mathrm{pH}$ varied from 5.0 to 7.4) were then examined. The PL intensity showed little fluctuation in the $\mathrm{pH}$ range from 5.0 to 6.8, while further increasing $\mathrm{pH}$ value led to slightly higher PL signal (Supplementary Fig. 41). Considering the acidic microenvironment of tumor site and the strong buffering capacity of PBS buffer, the influence of $\mathrm{pH}$ on PL signal in physiological conditions could be excluded. Therefore, it could be concluded that our Ppa-FFGYSA supermolecular 
probe could dynamically monitoring neutrophil infiltration and $\mathrm{ONOO}^{-}$production in real-time with high accuracy and sensitivity.

The feasibility of Ppa-FFGYSA to screen ICD drugs at the early stage of tumor immunogenicity transformation was firstly tested with hypericin (a commercial photosensitizer with strong ICD inducing capability) and the control drug cisplatin with weak ICD inducing capability. The drug-containing matrixgel was intratumorally administrated $12 \mathrm{~h}$ in advance, and hypericin group was then activated by light illumination $\left(0.4 \mathrm{~W} / \mathrm{cm}^{2}\right.$ for $\left.10 \mathrm{~min}\right)$ to induce ICD, followed by intratumoral injection of Ppa-FFGYSA at predesignated time points to monitor $\mathrm{ONOO}^{-}$levels with IVIS (Fig. 5f). The fluorescence signals at tumor sites were very consistent for each time point, confirming the same injection amount of Ppa-FFGYSA, and hence the PL signal changes shall be solely caused by ONOO- levels. The PL was rapidly activated and reached the maximum at $8 \mathrm{~h}$ post hypericin activation for hypericin group, and its intensity was much higher than that of the cisplatin group (an inefficient ICD inducer) for all the tested time points (Fig. 5g, h). There is negligible PL signal for control group without any drug treatment (Supplementary Fig. 42), indicating that the PL signal is activated by ICD drugs. The PL intensity for hypericin group was calculated to be $10.1 \times 10^{5} \mathrm{p} / \mathrm{s} / \mathrm{cm}^{2} / \mathrm{sr}$, which was 4.8-fold higher than cisplatin group and 81.9-fold higher than that of control group, suggesting the high sensitivity of our Ppa-FFGYSA probe towards ICD (Fig. 5i).

The infiltration of neutrophils during ICD process in the tumor was also examined by immunofluorescence staining. The gradually increase numbers of neutrophils suggests the tumor microenvironment switched from "cold" to "hot" upon hypericin activation. The degree of infiltrated neutrophils showed a good time-dependent correlation with the $\mathrm{ONOO}^{-}$activated PL, indicating that Ppa-FFGYSA probe serves well the purpose to monitor ICD drug activation (Supplementary Fig. 43). Comparison of infiltrated neutrophils for different groups was conducted at $8 \mathrm{~h}$ post drug activation. Hypericin group showed the highest neutrophil infiltration, which was 2.5-fold and 24.7-fold higher than that of cisplatin group and non-treated group, respectively, matching very well with PL results (Fig. 5j). The recruited CD8 ${ }^{+} \mathrm{T}$ cell level as the hallmark of tumor immunogenic environment, and the translation of ectocalreticulin (ecto-CRT) as the classical sign of ICD development were also evaluated. Hypericin group possesses 3.2-fold and 27.0-fold higher $\mathrm{CD} 8^{+} \mathrm{T}$ cell levels, and 4.9-fold and 33.8-fold higher ecto-CRT level than that of cisplatin group and non-treated group (Fig. 5j). These results suggested that the PL signal of Ppa-FFG-YSA could rapidly visualize the dynamic changes of $\mathrm{ONOO}^{-}$concentration in tumor cells induced by ICD drugs, revealing the process of tumor transformation from "cold" to "hot". It is worthy to note that the activated PL signal matches very well with the results from $\mathrm{CD} 8^{+} \mathrm{T}$ cells and ecto-CRT immunofluorescence methods, while our PL molecular probe does not require tedious staining procedures. Hence our Ppa-FFGYSA probe holds great advantage of high sensitivity, fast acquisition, and real- 
time manner. In addition, the Ppa-FFGYSA probe was also injected into the mice via tail vein immediately after ICD drug activation. Fluorescence imaging reveled the highest tumor accumulation at $8 \mathrm{~h}$ post injection, and the PL intensity also reached the maximum at the same time point, suggesting the feasibility of Ppa-FFGYSA to be used via tail vein injection for ICD drug screening (Supplementary Fig. 44a, b). Ex vivo imaging of isolated organs revealed that only drug treated-tumor showed PL signals although the probe was also distributed on other organs such as liver kidney and spleen as indicated by fluorescence imaging (Supplementary Fig. 41c). These results further reinforced the high specificity and sensitivity of Ppa-FFGYSA probe to be activated by ONOO" in the "hot" tumor induced by ICD drugs. Therefore, our PpaFFGYSA supermolecular probe represents the promising smart molecular NIR PL probes for screening of ICD drugs.

\section{Discussion}

We herein show that that porphyrin-derived organic photosensitizers with large heterocyclic structures, represented by Ppa, could achieve PL after cessation of excitation light or upon interaction with $\mathrm{ONOO}^{-}$. Mechanistic study found that the formation of unstable dioxetane intermediates from oxidization of vinylene bonds is the key step to realize PL, and the oxidization of vinylene bonds is an ordered process rather than a random ones, which was discovered for the first time. The ${ }^{1} \mathrm{O}_{2}$ production ability afforded porphyrin derivatives the selfsustainable PL after initiation. To the best of knowledge, Ppa has the longest emission (760 nm) of all the reported single-molecular based PL materials. PL imaging without excitation light and tissue autofluorescence interference possess great potential in translational research and clinical practice, but is limited by the lack of molecular probes with clinical potentials. Different from the current ones such as Cerenkov probes, rare-earth nanocrystals or semiconducting polymers, porphyrin derivatives have been widely applied in clinical practice. We demonstrated the PL of porphyrin derivatives and presented the rational explanation for such phenomenon, which should also be applicable to other porphyrin derivatives. Such discovery and mechanistic study provide a step forwards for developing porphyrin molecules and molecular imaging probes with NIR PL for translational research.

The Ppa-FFGYSA supermolecular probe we developed aims to increase the biocompatibility and imaging performance of Ppa. Ppa-FFGYSA formed ordered nanofibrous $\beta$-sheet structures, which promote photoacoustic and PL signals for preoperative photoacoustic imaging and intraoperative PL image-guided tumor resection. ${ }^{57}$ Such PL could last over $60 \mathrm{~min}$ upon single activation, and could be repeatedly activated for over 10 cycles, providing sufficient time for cancer surgery. The PL is more favorable for intraoperative imaging as compared to fluorescence as it eliminates the inference from real-time photoexcitation and therefore has a 
SBR that is over 50-fold higher than fluorescence imaging (3235.3 versus 76.2). To this end, Ppa-FFGYSA was successfully applied for precise resection of orthotopic breast cancer and peritoneal metastases under PL image guidance. As for image-guided cancer surgery, the high intraoperative SBRs (215.1 and 193.1 for two demonstrated models) provided by PL images are much higher than that of reported fluorescence-based ones, ${ }^{6,5}, 59$, which enabled complete tumor nodule resection and improved surgery outcomes largely. The specific responsiveness of Ppa towards $\mathrm{ONOO}^{-}$also permits the development of smart activable PL molecular probes for real-time monitoring $\mathrm{ONOO}^{-}$dynamics, which provided fast and accurate accessing of neutrophil infiltration in vivo. Furthermore, Ppa-FFGYSA also permits the rapid and sensitive screening of ICD drugs in living mice, which is faster and more convenient than traditional histological immunofluorescence analysis including neutrophils, CD8+ T cells, and ecto-CRT.

In conclusion, we have explored and screened porphyrin derivatives as PL molecular probes for in vivo optical imaging applications for the first time. The PL origin of porphyrin molecules was unveiled with a plausible explanation using Ppa as the example, which provides the mechanism foundation for their PL (afterglow and chemiluminescence) applications. PpaFFGYSA $\beta$-sheet supramolecular aggregates were further constructed with enhanced photoacoustic and PL signals, active tumor targeting capability. The long lasting PL without real-time excitation light interference enables precise image-guided tumor surgery and sensitive screening of ICD drugs in living mice. Considering various porphyrin derivatives have been approved for clinical practices, and the vast availability of optical imaging instruments, our discovery and demonstration further illustrate their great promise as advanced PL imaging probes over fluorescence ones in clinical applications.

\section{Materials and Methods}

Materials. All porphyrin derivative molecules were purchased from Sigma-Aldrich unless otherwise stated. The co-polymer lipid-PEG ${ }_{2000}$ (1,2-distearoyl-sn-glycero-3phosphoethanolamine-N-[methoxy(polyethylene glycol)-2000]) was purchased from Laysan Bio, Inc. (Arab, AL). BD Matrigel ${ }^{\mathrm{TM}}$ was purchased from BD company. 2-Cl-trityl chloride resin (1.0-1.2 $\mathrm{mmol} \mathrm{g}^{-1}$ ) and other Fmoc-amino acids were purchased from GL Biochem (Shanghai, China). Dulbecco's Modified Eagle's Medium (DMEM), fetal bovine serum (FBS) and penicillin-streptomycin were provided by Thermo Fisher Scientific Inc. (Waltham, MA, USA). Monoclonal anti-EphA2 antibody and anti-calreticulin antibody were purchased from Abcam and Alexa Fluor $488^{\circledR}$-conjugated secondary antibody was purchased from Life Technologies. Antibodies for neutrophils and $\mathrm{CD}^{+} \mathrm{T}$ cell immunofluorescent staining were available from Biolegend.

Characterization. The UPLC-HRMS analysis was conducted on ACQUITY UPLC I- 
Class/UPCC/M Class/SYNAPT G2-SI. The synthesized peptide molecules were isolated and purified by LUMTECH HPLC (Germany) system using a C18 RP column with $\mathrm{MeOH}$ and water containing $0.1 \%$ of TFA as the eluents. The structures of the synthesized products was determined by LCMS-20AD (Shimadzu) system, Bruker AutoflexIII LRF200-CID mass spectrometer in matrix-assisted laser desorption ionization time-of-flight (MALDI-TOF) mode. NMR spectra were recorded on a Bruker ARX 400 NMR spectrometer. UV-vis absorption spectra and fluorescence spectra were recorded on Shimadzu UV-1700 spectrometer and Perkin-Elmer LS-55 spectrometer, respectively. The values of kcps were recorded by dynamic light scattering (DLS) at a fixed angle of $90^{\circ}$ at room temperature using a particle size analyzer (90 Plus, Brookhaven Instruments Co) to obtain the critical micelle concentrations (CMCs). Then transmission electron microscopy (TEM, JEM-2010F, JEOL, Japan) was used to determine the morphology. Circular dichroism (CD) spectrum subtracting the solvent background was measured by a BioLogic (MOS-450) system. Fluorescence imaging of cell and tissue sections were performed by confocal laser scanning microscopy (CLSM, Zeiss LSM 710, Jena, Germany). Fluorescence and PL images were acquired on an in vivo imaging system (IVIS Spectrum, PerkinElmer). Photoacoustic imaging was performed by a commercial smallanimal opt-acoustic tomography system (MOST, iTheraMedical, Germany).

Synthesis of Ppa-FFGYSA and Ppa-YSA. According to our previous work ${ }^{60}$, a standard solidphase peptide synthesis method was used to yield amino-group-bearing peptides $\mathrm{NH}_{2}$-FFGYSA and $\mathrm{NH}_{2}$-YSA. In details, to the DCM solution of Ppa (10 mg), N-hydroxysuccinimide (NHS, $7.7 \mathrm{mg}$ ) and dicyclohexylcarbodiimide (DCC, $4.3 \mathrm{mg}$ ) were added, the solution was then stirred in dark for $3 \mathrm{~h}$ to activate the carboxyl group of Ppa. The precipitate was removed with a filter membrane, and the supernatant was collected and evaporated to dryness for subsequent use. Excess $\mathrm{NH}_{2}$-FFGYSA (50 mg) were then dissolved in DMF and $\mathrm{pH}$ was adjusted to 8-9 with DIPEA buffer $(25 \mu \mathrm{L})$. Ppa was them added and the mixture was stirred overnight at room temperature. The final product was purified by HPLC, and the structure was confirmed by LCMS and ${ }^{1} \mathrm{H}$ NMR. The synthesis method of Ppa-YSA was similar to that of Ppa-FFGYSA.

Ppa-FFGYSA: ${ }^{1} \mathrm{H}$ NMR (400 MHz, DMSO) $\delta 9.72(\mathrm{~s}, 1 \mathrm{H}), 9.43$ (s, 1H), 8.89 (s, 1H), $8.31-$ $7.55(\mathrm{~m}, 13 \mathrm{H}), 7.37-6.89(\mathrm{~m}, 15 \mathrm{H}), 6.76(\mathrm{t}, J=7.3 \mathrm{~Hz}, 1 \mathrm{H}), 6.62(\mathrm{dd}, J=15.1,8.4 \mathrm{~Hz}, 4 \mathrm{H})$, $6.39(\mathrm{~d}, J=17.9 \mathrm{~Hz}, 1 \mathrm{H}), 6.21(\mathrm{~d}, J=11.6 \mathrm{~Hz}, 1 \mathrm{H}), 5.09(\mathrm{dd}, J=38.6,20.1 \mathrm{~Hz}, 2 \mathrm{H}), 4.71-$ $4.14(\mathrm{~m}, 18 \mathrm{H}), 3.89-3.53(\mathrm{~m}, 20 \mathrm{H}), 3.21(\mathrm{~s}, 3 \mathrm{H}), 2.97-2.59(\mathrm{~m}, 12 \mathrm{H}), 2.40-2.17(\mathrm{~m}, 3 \mathrm{H})$, $2.15-1.69(\mathrm{~m}, 21 \mathrm{H}), 1.62(\mathrm{t}, J=7.5 \mathrm{~Hz}, 4 \mathrm{H}), 1.32-1.05(\mathrm{~m}, 10 \mathrm{H}), 0.94-0.73(\mathrm{~m}, 7 \mathrm{H}), 0.24$ (s, 1H). MALDI-TOF MS: $\mathrm{M}+\mathrm{Na}^{+}$found 2236.93.

Ppa-YSA: ${ }^{1} \mathrm{H}$ NMR (400 MHz, DMSO) $\delta 9.74$ (s, 1H), 9.46 (s, 1H), 8.89 (s, 1H), $8.47-7.42$ (m, 10H), $7.08-6.87(\mathrm{~m}, 4 \mathrm{H}), 6.60(\mathrm{t}, J=8.6 \mathrm{~Hz}, 4 \mathrm{H}), 6.40(\mathrm{~d}, J=18.0 \mathrm{~Hz}, 1 \mathrm{H}), 6.22(\mathrm{~d}, J=$ $11.8 \mathrm{~Hz}, 1 \mathrm{H}), 5.14(\mathrm{dd}, J=55.1,19.9 \mathrm{~Hz}, 2 \mathrm{H}), 4.61-4.11(\mathrm{~m}, 15 \mathrm{H}), 3.84-3.59$ (m, 12H), 
$3.45(\mathrm{~s}, 3 \mathrm{H}), 3.23(\mathrm{~s}, 3 \mathrm{H}), 2.94-2.81(\mathrm{~m}, 2 \mathrm{H}), 2.77-2.52(\mathrm{~m}, 4 \mathrm{H}), 2.47-2.28(\mathrm{~m}, 7 \mathrm{H}), 2.08$ $-1.69(\mathrm{~m}, 24 \mathrm{H}), 1.63(\mathrm{t}, J=7.5 \mathrm{~Hz}, 3 \mathrm{H}), 1.35-0.98(\mathrm{~m}, 11 \mathrm{H}), 0.95-0.66(\mathrm{~m}, 8 \mathrm{H}), 0.26(\mathrm{~s}$, 1H). MALDI-TOF MS: M+H ${ }^{+}$found 1863.77.

In vitro fluorescence and PL imaging. An IVIS imaging system was used for fluorescence and PL imaging (with or without excitation mode), including the acquisition of PL spectra. For in vitro and in vivo fluorescence imaging, images were acquired with excitation at $675 \mathrm{~nm}$, and emission at $720 \mathrm{~nm}$ unless otherwise stated. For acquisition of PL images, samples were preirradiated by $660 \mathrm{~nm}$ laser for $2 \mathrm{~min}$ at a power of $1.4 \mathrm{~W} / \mathrm{cm}^{2}$, or mixed with $\mathrm{ONOO}^{-}$or other RONSs $(80 \mu \mathrm{M})$, and then the PL images were acquired with an exposure time of $60 \mathrm{~s}$ using an open filter or a specific wavelength emission filter. The fluorescence or PL data were quantified with ROI analysis using Living Image 4.0 Software.

Cell culture and imaging. The murine 4T1 breast cancer cells and human smooth muscle cells (SMCs) were purchased from American Type Culture Collection (ATCC). Cells were grown in DMEM medium containing $10 \%$ fetal bovine serum (FBS) and $1 \%$ penicillin-streptomycin at $37{ }^{\circ} \mathrm{C}$ in a humidified environment containing $5 \% \mathrm{CO}_{2}$ and regularly checked for mycoplasma contamination. For cell imaging, 4T1 cancer cells and SMCs cells were seeded in confocal dishes and then grown for $24 \mathrm{~h}$. Then $2 \mu \mathrm{M}$ of Ppa-FFGYSA or Ppa-YSA in serum-free cell culture medium were added and incubated for $2 \mathrm{~h}$ at $37^{\circ} \mathrm{C}$, followed by washing with $1 \times \mathrm{PBS}$ buffer for three times, and fixing with $4 \%$ paraformaldehyde for $10 \mathrm{~min}$. The cells were incubated with 4',6-diamidino-2-phenylindole (DAPI) for $15 \mathrm{~min}$ to stain the nuclei, and then imaged by CLSM after washing with $1 \times$ PBS buffer. For co-staining with anti-EphA $\mathrm{A}_{2}$ antibody, the Ppa-FFGYSA or Ppa-YSA treated 4T1 cells were incubated with anti-EphA 2 antibody overnight at $4^{\circ} \mathrm{C}$. Subsequently, the cells were rewarmed for $1 \mathrm{~h}$ and further incubated with AlexaFluor $^{\circledR} 488$-conjugated second antibody $(10 \mu \mathrm{g} / \mathrm{mL})$ for $90 \mathrm{~min}$ and DAPI for $15 \mathrm{~min}$ in sequence, followed by imaging with CLSM. For blocking experiment, the 4T1 cells were pretreated with an excess of free YSAYPDSVPMMS peptides $(500 \mu \mathrm{M})$ to seal the binding site of EphA $\mathrm{A}_{2}$ protein, and then incubated with $2 \mu \mathrm{M}$ of Ppa-FFGYSA or Ppa-YSA at $37^{\circ} \mathrm{C}$ for $2 \mathrm{~h}$, followed by CLSM imaging under the same conditions as described above.

Animals and tumor-bearing mouse model. All animal studies were performed according to the guidelines set by the Tianjin Committee of Use and Care of Laboratory Animals, and the overall project protocols were approved by the Animal Ethics Committee of Nankai University. The accreditation number of the laboratory is SYXK (Jin) 2019-0003 promulgated by Tianjin Science and Technology Commission. The female BALB/c mice were purchased from Laboratory Animal Center of the Academy of Military Medical Sciences (Beijing, China). $1 \times$ $10^{6} 4 \mathrm{~T} 1$ breast cancer cells in $50 \mu \mathrm{L}$ PBS were subcutaneously injected into the second breast fat pad on right side to establish the orthotopic $4 \mathrm{~T} 1$ breast tumor-bearing mouse mode. The 
mouse was used for subsequent fluorescence, PL and photoacoustic imaging when the tumor volume reaches $80 \mathrm{~mm}^{3}$. For peritoneal carcinomatosis-bearing mouse model, same amount of $4 \mathrm{~T} 1$ cancer cells were intraperitoneally injected into the BALB/c mice, which were used for imaging or surgical excision after approximately one week.

In vivo photoacoustic imaging. In vivo photoacoustic imaging of tumors was performed by a commercial small-animal opt-acoustic tomography system (MOST, iTheraMedical, Germany). The established orthotopic $4 \mathrm{~T} 1$ breast tumor-bearing female mice were grouped $(n=3)$ and then injected with Ppa-FFGYSA or Ppa-YSA (500 $\mu$ M, $200 \mu \mathrm{L}$, PBS:DMSO=95:5, v/v) through the tail vein, and then the photoacoustic signals of the tumors were acquired at several time intervals before and after the injection of these probes. The mice were anesthetized with isoflurane in oxygen and placed in an aqueous environment with the tumor areas coated with ultrasonic coupling agent before photoacoustic imaging. The final photoacoustic signal was calculated by subtracting from the signal of $\mathrm{Hb}$ and $\mathrm{HbO}_{2}$. For peritoneal carcinomatosisbearing mice models, the PA images were acquired before administration and at designated time intervals after injection.

Tumor resection and intraoperative PL imaging. The orthotopic 4T1 breast tumor-bearing mouse were selected and intravenously injected with $200 \mu \mathrm{L}$ of Ppa-FFGYSA probe (500 $\mu \mathrm{M}$, PBS:DMSO=95:5, v/v). The Xenogen IVIS ${ }^{\circledR}$ Lumina II system was used for subsequent experiment. With the information provided by photoacoustic imaging, surgery was performed at $8 \mathrm{~h}$ post injection. The mice were anesthetized with isoflurane in the induction chamber and the PL signal was acquired immediately with an open filter for $60 \mathrm{~s}$ after laser pre-irradiation of the tumor tissue $\left(660 \mathrm{~nm}, 1.4 \mathrm{~W} / \mathrm{cm}^{2}, 2 \mathrm{~min}\right)$. The fluorescence signal was acquired with an excitation at $675 \mathrm{~nm}$ and an emission wavelength of $720 \mathrm{~nm}$. The tumor was then excised using a sterile scalpel under the guidance of PL imaging. The excised tissues were then fixed, dehydrated, embedded, sectioned, and stained with $H \& E$. H\&E slices were observed under a digital microscope (Leica QWin) to illustrate the accuracy of the surgical excision. For surgical resection of abdominal tumors, peritoneal carcinomatosis-bearing mouse models were injected with Ppa-FFGYSA through the tail vein. The mice were anesthetized and then the abdominal skin of the mice was cut with prior to surgery at $24 \mathrm{~h}$ post injection, followed by laser irradiation to activate the PL signal. The tumor nodules were removed effectively based on the PL imaging without the surgeon's assistance, and the removed tumor nodules were imaged and analyzed by fluorescence and PL imaging, followed by histological staining analysis. The imaging strategy for the entire procedure was as for the orthotopic tumor resection procedure.

Monitoring of neutrophil infiltration. LPS was used to induce neutrophil infiltration at skin. $50 \mu \mathrm{g}$ of LPS was dissolved in $25 \mu \mathrm{L}$ water and then injected subcutaneously into the dorsal location near the hind limb of the mice. Subsequently, Ppa-FFGYSA probe $(500 \mu \mathrm{M}, 50 \mathrm{uL}$ 
dissolved in PBS) were subcutaneously injected into the LPS site at predesignated time intervals $(0,0.17,0.5,1,2,4,8$ and $24 \mathrm{~h}$, respectively). The FL and PL images were then acquired using IVIS system.

Induction and monitoring of ICD response. The orthotopic 4T1 breast tumor-bearing mouse were randomly divided into 3 groups: control, cisplatin and hypericin group. Matrigel mixed with hypericin $(10 \mu \mathrm{g} / \mathrm{mL}, 25 \mu \mathrm{L})$ or cisplatin $(600 \mu \mathrm{g} / \mathrm{mL}, 30 \mu \mathrm{L})$ were intratumorally injected into the tumors. After $12 \mathrm{~h}$ of intratumoral administration, the hypericin group was locally irradiated with white light irradiation $\left(0.4 \mathrm{~W} / \mathrm{cm}^{2}, 10 \mathrm{~min}\right)$. The Ppa-FFGYSA probe was then injected intratumorally at $0,2.5,5,8$ and $24 \mathrm{~h}$ post hypericin activation. The FL and PL images were immediately acquired with IVIS system after probe injection. The Ppa-FFGYSA probe was also intravenously injection into the mouse through tail vein immediately after hypericin activation, and the FL and PL images were acquired thereafter. For ex vivo imaging, the major organs of the mice (including heart, liver, spleen, lung and kidney) were then extracted at $8 \mathrm{~h}$ post Ppa-FFGYSA injection, and subjected to IVIS imaging. The imaging protocol was as described above.

Immunofluorescence staining. The skin tissues were extracted at predesignated time intervals post LPS injection and fixed. The tissues were then dehydrated with sucrose solutions of different concentration gradients $(20 \%, 30 \%, 40 \%)$ and were immersed in optimum cutting temperature compound (OCT) and frozen at $-20^{\circ} \mathrm{C}$. The frozen tissues were then cut into $6 \mu \mathrm{m}$ slices and stained for immunofluorescence. In details, tissue slices were fixed in cold acetone for 10 minutes and blocked with goat serum for 30 minutes at room temperature to avoid nonspecificity. Excess goat serum was wiped away and the slice was stained with antibody (neutrophils) for $2 \mathrm{~h}$ at room temperature. Excess antibody was then washed away with PBS buffer, followed by sealing and staining of nuclei with DAPI solution. The stained slices were stored in the dark for subsequent CLSM imaging. A very similar procedure was applied for tumor tissues receiving ICD drugs. In details, tumor tissues were extracted at predesignated time intervals post ICD drug treatment, and then fixed, dehydrated, immersed in OCT, froze, and sectioned into slices. Immunofluorescence staining and imaging of neutrophils and CD8 ${ }^{+}$ $\mathrm{T}$ cells were performed as above. For calreticulin staining, the tumor tissues were stained with anti-calreticulin antibody (1:200 dilution, 1× PBS) overnight, AlexaFluor ${ }^{\circledR}$-conjugated secondary antibody (1:200 dilution, $1 \times \mathrm{PBS})$ for $2 \mathrm{~h}$ at room temperature, and DAPI-containing block solution in sequence. CLSM imaging was then performed to visualize ecto-CRT expression in each group.

Statistical Analysis. Quantitative data were expressed as mean \pm standard deviation (SD). Statistical comparisons were made by ANOVA analysis and two-sample Student's t-test. $P$ value $<0.05$ was considered statistically significant. 


\section{Data availability}

The authors declare that all data from this study are available within the Article and its Supplementary Information. Raw data for the individual measurements are available on reasonable request.

\section{References}

1. Choi, H.S. et al. Targeted zwitterionic near-infrared fluorophores for improved optical imaging. Nat. Biotechnol. 31, 148-153 (2013).

2. Vahrmeijer, A.L., Hutteman, M., van der Vorst, J.R., van de Velde, C.J.H. \& Frangioni, J.V. Imageguided cancer surgery using near-infrared fluorescence. Nat. Rev. Clin. Oncol. 10, 507-518 (2013).

3. Kobayashi, H., Ogawa, M., Alford, R., Choyke, P.L. \& Urano, Y. New Strategies for Fluorescent Probe Design in Medical Diagnostic Imaging. Chem. Rev. 110, 2620-2640 (2010).

4. Pan, Y. et al. Endoscopic molecular imaging of human bladder cancer using a CD47 antibody. Sci. Transl. Med. 6, 260ra148-260ra148 (2014).

5. Koller, M. et al. Implementation and benchmarking of a novel analytical framework to clinically evaluate tumor-specific fluorescent tracers. Nat. Commun. 9, 3739 (2018).

6. Hu, Z. et al. First-in-human liver-tumour surgery guided by multispectral fluorescence imaging in the visible and near-infrared-I/II windows. Nat. Biomed. Eng. 4, 259-271 (2020).

7. Luby, B.M., Charron, D.M., MacLaughlin, C.M. \& Zheng, G. Activatable fluorescence: From small molecule to nanoparticle. Adv. Drug Delivery Rev. 113, 97-121 (2017).

8. Huang, J., Li, J., Lyu, Y., Miao, Q. \& Pu, K. Molecular optical imaging probes for early diagnosis of drug-induced acute kidney injury. Nat. Mater. 18, 1133-1143 (2019).

9. van Manen, L. et al. A practical guide for the use of indocyanine green and methylene blue in fluorescence-guided abdominal surgery. J. Surg. Oncol. 118, 283-300 (2018).

10. Rosenthal, E.L. et al. Sensitivity and Specificity of Cetuximab-IRDye800CW to Identify Regional Metastatic Disease in Head and Neck Cancer. Clin. Cancer Res. 23, 4744-4752 (2017).

11. van Dam, G.M. et al. Intraoperative tumor-specific fluorescence imaging in ovarian cancer by folate receptor- $\alpha$ targeting: first in-human results. Nat. Med. 17, 1315-1319 (2011).

12. Liu, R., Xu, Y., Xu, K. \& Dai, Z. Current trends and key considerations in the clinical translation of targeted fluorescent probes for intraoperative navigation. Aggregate 2 (2021).

13. Kenry, Duan, Y. \& Liu, B. Recent Advances of Optical Imaging in the Second Near-Infrared Window. Adv. Mater. 30, 1802394 (2018).

14. Sun, C. et al. J-Aggregates of Cyanine Dye for NIR-II in Vivo Dynamic Vascular Imaging beyond 1500 nm. J. Am. Chem. Soc. 141, 19221-19225 (2019).

15. Yang, Q. et al. Donor Engineering for NIR-II Molecular Fluorophores with Enhanced Fluorescent 
Performance. J. Am. Chem. Soc. 140, 1715-1724 (2018).

16. Antaris, A.L. et al. A small-molecule dye for NIR-II imaging. Nat. Mater. 15, 235-242 (2016).

17. Helmchen, F. \& Denk, W. Deep tissue two-photon microscopy. Nat. Methods 2, 932-940 (2005).

18. Chen, S. et al. Near-infrared deep brain stimulation via upconversion nanoparticle-mediated optogenetics. Science 359, 679-684 (2018).

19. Cai, Y. et al. Optical nano-agents in the second near-infrared window for biomedical applications. Chem. Soc. Rev. 48, 22-37 (2019).

20. Hong, G., Antaris, A.L. \& Dai, H. Near-infrared fluorophores for biomedical imaging. Nat. Biomed. Eng. 1, 0010 (2017).

21. So, M.-K., Xu, C., Loening, A.M., Gambhir, S.S. \& Rao, J. Self-illuminating quantum dot conjugates for in vivo imaging. Nat. Biotechnol. 24, 339-343 (2006).

22. Hananya, N. \& Shabat, D. A Glowing Trajectory between Bio- and Chemiluminescence: From Luciferin-Based Probes to Triggerable Dioxetanes. Angew. Chem. Int. Ed. 56, 16454-16463 (2017).

23. Liu, J. et al. Imaging and therapeutic applications of persistent luminescence nanomaterials. $A d v$. Drug Delivery Rev. 138, 193-210 (2019).

24. Xu, S., Chen, R., Zheng, C. \& Huang, W. Excited State Modulation for Organic Afterglow: Materials and Applications. Adv. Mater. 28, 9920-9940 (2016).

25. Suzuki, K. \& Nagai, T. Recent progress in expanding the chemiluminescent toolbox for bioimaging. Curr. Opin. Biotechnol. 48, 135-141 (2017).

26. Ruggiero, A., Holland, J.P., Lewis, J.S. \& Grimm, J. Cerenkov Luminescence Imaging of Medical Isotopes. J. Nucl. Med. 51, 1123-1130 (2010).

27. Liu, H. et al. Intraoperative Imaging of Tumors Using Cerenkov Luminescence Endoscopy: A Feasibility Experimental Study. J. Nucl. Med. 53, 1579-1584 (2012).

28. Pei, P. et al. X-ray-activated persistent luminescence nanomaterials for NIR-II imaging. Nat. Nanotechnol. 16, 1011-1018 (2021).

29. Maldiney, T. et al. The in vivo activation of persistent nanophosphors for optical imaging of vascularization, tumours and grafted cells. Nat. Mater. 13, 418-426 (2014).

30. Sun, S.-K., Wang, H.-F. \& Yan, X.-P. Engineering Persistent Luminescence Nanoparticles for Biological Applications: From Biosensing/Bioimaging to Theranostics. Acc. Chem. Res. 51, 11311143 (2018).

31. Green, O. et al. Opening a Gateway for Chemiluminescence Cell Imaging: Distinctive Methodology for Design of Bright Chemiluminescent Dioxetane Probes. ACS Cent. Sci 3, 349-358 (2017).

32. Miao, Q. et al. Molecular afterglow imaging with bright, biodegradable polymer nanoparticles. Nat. Biotechnol. 35, 1102-1110 (2017).

33. Jiang, Y. et al. A generic approach towards afterglow luminescent nanoparticles for ultrasensitive in vivo imaging. Nat. Commun. 10, 2064 (2019).

34. Lu, L. et al. NIR-II bioluminescence for in vivo high contrast imaging and in situ ATP-mediated metastases tracing. Nat. Commun. 11, 4192 (2020). 
35. Ni, X.; Zhang, X.; Duan, X.; Zheng, H.-L.; Xue, X.-S.; Ding, D. Near-infrared afterglow luminescent aggregation-induced emission dots with ultrahigh tumor-to-liver signal ratio for promoted image-guided cancer surgery. Nano Lett. 19, 318-330 (2019).

36. Adler-Abramovich, L. \& Gazit, E. The physical properties of supramolecular peptide assemblies: from building block association to technological applications. Chem. Soc. Rev. 43, 6881-6893 (2014).

37. Greenfield, N.J. Using circular dichroism spectra to estimate protein secondary structure. Nat. Protoc. 1, 2876-2890 (2006).

38. Zhang, L. et al. Transformable peptide nanoparticles arrest HER2 signalling and cause cancer cell death in vivo. Nat. Nanotechnol. 15, 145-153 (2020).

39. Ogi, S., Sugiyasu, K., Manna, S., Samitsu, S. \& Takeuchi, M. Living supramolecular polymerization realized through a biomimetic approach. Nat. Chem. 6, 188-195 (2014).

40. Li, L.L. et al. Pathological-Condition-Driven Construction of Supramolecular Nanoassemblies for Bacterial Infection Detection. Adv. Mater. 28, 254-262 (2016).

41. Matile, S., Berova, N., Nakanishi, K., Fleischhauer, J. \& Woody, R.W. Structural Studies by Exciton Coupled Circular Dichroism over a Large Distance: Porphyrin Derivatives of Steroids, Dimeric Steroids, and Brevetoxin B. J. Am. Chem. Soc. 118, 5198-5206 (1996).

42. Fabiano, A.-S., Allouche, D., Sanejouand, Y.-H. \& Paillous, N. Synthesis of a New Cationic Pyropheophorbide Derivative and Studies of its Aggregation Process in Aqueous Solution. Photochem. Photobiol. 66, 336-345 (1997).

43. Miller, M., Gillbro, T. \& Olson, J.M. AQUEOUS AGGREGATES OF BACTERIOCHLOROPHYLL c AS A MODEL FOR PIGMENT ORGANIZATION IN CHLOROSOMES. Photochem. Photobiol. 57, 98-102 (1993).

44. Feng, G., Zhang, G.-Q. \& Ding, D. Design of superior phototheranostic agents guided by Jablonski diagrams. Chem. Soc. Rev. 49, 8179-8234 (2020).

45. Weber, J., Beard, P.C. \& Bohndiek, S.E. Contrast agents for molecular photoacoustic imaging. Nat. Methods 13, 639-650 (2016).

46. Zhang, D. et al. In Situ Formation of Nanofibers from Purpurin18-Peptide Conjugates and the Assembly Induced Retention Effect in Tumor Sites. Adv. Mater. 27, 6125-6130 (2015).

47. Qi, J. et al. Light-driven transformable optical agent with adaptive functions for boosting cancer surgery outcomes. Nat. Commun. 9, 1848 (2018).

48. Wang, W. et al. Molecular Cancer Imaging in the Second Near-Infrared Window Using a RenalExcreted NIR-II Fluorophore-Peptide Probe. Adv. Mater. 30, 1800106 (2018).

49. Yang, J. et al. Turn-on chemiluminescence probes and dual-amplification of signal for detection of amyloid beta species in vivo. Nat. Commun. 11, 4052 (2020).

50. Yan, R. et al. Activatable NIR Fluorescence/MRI Bimodal Probes for in Vivo Imaging by EnzymeMediated Fluorogenic Reaction and Self-Assembly. J. Am. Chem. Soc. 141, 10331-10341 (2019).

51. Wang, H., Feng, Z., Del Signore, S.J., Rodal, A.A. \& Xu, B. Active Probes for Imaging Membrane 
Dynamics of Live Cells with High Spatial and Temporal Resolution over Extended Time Scales and Areas. J. Am. Chem. Soc. 140, 3505-3509 (2018).

52. Garg, A.D. et al. Pathogen response-like recruitment and activation of neutrophils by sterile immunogenic dying cells drives neutrophil-mediated residual cell killing. Cell Death Differ. 24, 832-843 (2017).

53. Engeman, T., Gorbachev, A.V., Kish, D.D. \& Fairchild, R.L. The intensity of neutrophil infiltration controls the number of antigen-primed CD8 T cells recruited into cutaneous antigen challenge sites. J. of Leukocyte Biol. 76, 941-949 (2004).

54. Kolaczkowska, E. \& Kubes, P. Neutrophil recruitment and function in health and inflammation. Nat. Rev. Immunol. 13, 159-175 (2013).

55. Song, Z. et al. Activatable Fluorescent Nanoprobe with Aggregation-Induced Emission Characteristics for Selective In Vivo Imaging of Elevated Peroxynitrite Generation. Adv. Mater. 28, 7249-7256 (2016).

56. Eiserich, J.P. et al. Formation of nitric oxide-derived inflammatory oxidants by myeloperoxidase in neutrophils. Nature 391, 393-397 (1998).

57. Qi, J. et al. Light-driven transformable optical agent with adaptive functions for boosting cancer surgery outcomes. Nat. Commun. 9, 1848 (2018).

58. Zhu, S., Tian, R., Antaris, A.L., Chen, X. \& Dai, H. Near-Infrared-II Molecular Dyes for Cancer Imaging and Surgery. Adv. Mater. 31, 1900321 (2019).

59. Sun, Y. et al. Novel bright-emission small-molecule NIR-II fluorophores for in vivo tumor imaging and image-guided surgery. Chem. Sci. 8, 3489-3493 (2017).

60. Chen, C. et al. AIEgen-based theranostic system: targeted imaging of cancer cells and adjuvant amplification of antitumor efficacy of paclitaxel. Chem. Sci. 8, 2191-2198 (2017).

\section{Acknowledgements}

This work was supported by the NSFC (51961160730, 51873092, and 81901874), the National Key R\&D Program of China (Intergovernmental Cooperation Project, 2017YFE0132200), the Fundamental Research Funds for the Central Universities, and the Tianjin Science Fund for Distinguished Young Scholars (19JCJQJC61200).

\section{Author contributions}

D.D. and G.F. conceived the idea of the project. X.D. conducted all experiments and analyzed data. G.-Q.Z. assisted with mechanistic studies. S.J. performed image post-processing. X.D. and Y.Z. performed the in vivo experiments. H.O. and Z.G. provided assistance with in vivo imaging. J.L. assisted with peptide synthesis. D.D., G.F., X.D. and G.-Q.Z. wrote the paper and all authors participated in the discussion of the manuscript. D.D. supervised the whole project. X.D. and G.-Q. Z. contributed equally. 


\section{Competing interests}

The authors declare no competing interests. 